\title{
Satisfação dos familiares com os serviços de saúde mental para crianças e adolescentes: uma revisão sistemática
}

\author{
Family satisfaction with mental health services \\ for children and adolescents: a systematic review
}

\author{
Daianna Lima Thiengo', Diego da Fonseca², Giovanni Marcos Lovisi ${ }^{3}$
}

\begin{abstract}
Resumo
Introdução: A avaliação dos familiares sobre a satisfação com os serviços de saúde mental para crianças e adolescentes pode ser particularmente útil, pois tais serviços são os principais provedores de cuidados, o que afeta os resultados do tratamento. O objetivo do presente estudo foi realizar uma revisão sistemática sobre a satisfação entre familiares com os serviços de saúde mental infantojuvenil, assim como analisar os fatores associados. Métodos: Busca de artigos publicados nas bases de dados PubMed/MEDLINE, ScienceDirect, Web of Science, CAPES Periódicos, LILACS/BIREME, Scopus e PsycINFO entre 1994 e 2014. Foram considerados critérios de inclusão: estudos epidemiológicos observacionais; com instrumentos validados; publicados em inglês, espanhol ou português; e que obtiveram pontuação acima de 12 pontos conforme critérios metodológicos do Checklist for Measuring Quality. Resultados: Em todos os estudos, os familiares estavam satisfeitos com os serviços de atendimento. Os principais fatores associados foram: menor gravidade dos sintomas, maior idade do paciente, maior frequência no serviço e diagnóstico de retardo mental, qualidade no atendimento individual, presença de atendimento familiar, menor nível educacional e cor branca dos familiares. Conclusão: Programas de assistência devem ficar atentos às medidas de satisfação, destacando a necessidade da integração da assistência em saúde mental com o familiar. O conhecimento dessa temática torna-se fundamental para a implantação de melhorias nos programas assistenciais.
\end{abstract}

Palavras-chave: satisfação dos consumidores; cuidadores familiares; crianças; adolescentes; serviços de saúde mental.

\begin{abstract}
Introduction: The evaluation of family members on satisfaction with mental health services for children and adolescents can be particularly useful, since they are the main providers of care affecting treatment outcomes. The objective was to conduct a systematic review of satisfaction among family members with services of infantojuvenil mental health as well as to analyze associated factors. Methods: Search articles published in the PubMed/MEDLINE, ScienceDirect, Web of Science, CAPES Periódicos, LILACS/BIREME, Scopus and PsycINFO data between 1994 and 2014. Inclusion criteria were: observational epidemiological studies, with validated instruments; published in English, Spanish or Portuguese, and who achieved a score above 12 points as methodological criteria of the Checklist for Measuring Quality. Results: In all studies, the relatives were satisfied with customer services. The associated factors were less severe symptoms, greater patient age, more often in the service and diagnosis of mental retardation, quality individual care, presence of family care, lower educational level and the white family. Conclusion: Assistance programs beware measures of satisfaction, highlighting the need for integration of mental health care with the family. The knowledge of this issue becomes critical to implementation of improvements in welfare programs.
\end{abstract}

Keywords: consumer satisfaction; family caregivers; children; adolescent; mental health services.

Trabalho realizado no Instituto de Estudos em Saúde Coletiva da Universidade Federal do Rio de Janeiro (UFRJ) - Rio de Janeiro (RJ), Brasil. `Doutoranda em Saúde Coletiva pelo Instituto de Estudos em Saúde Coletiva da UFRJ - Rio de Janeiro (RJ), Brasil.

${ }^{2}$ Doutorando em Saúde Coletiva pelo Instituto de Estudos em Saúde Coletiva da UFRJ - Rio de Janeiro (RJ), Brasil.

${ }_{3}^{3}$ Professor Associado do Instituto de Estudos em Saúde Coletiva da UFRJ - Rio de Janeiro (RJ), Brasil.

Endereço para correspondência: Daianna Lima Thiengo - Avenida Doutor Carvalhaes, 1.048, casa - Rocha Sobrinho - CEP: 26572-530 - Mesquita (RJ), Brasil -E-mail: daianna.thiengo@yahoo.com.br

Fonte de financiamento: Fundação Carlos Chagas de Amparo à Pesquisa do Estado do Rio de Janeiro (FAPERJ - E-26/111.827/2012) e Coordenação de Aperfeiçoamento de Pessoal de Nível Superior (CAPES) e Conselho Nacional de Desenvolvimento Científico e Tecnológico (CNPq) (470217/2012-0). Conflito de interesses: nada a declarar. 


\section{INTRODUÇÃO}

Segundo a Organização Mundial da Saúde (OMS) ${ }^{1}$, a faixa etária que compreende a infância vai de 0 a 9 anos de idade, enquanto a adolescência compreende a faixa etária entre 10 e 19 anos. Nessas populações são encontradas altas proporções de transtornos mentais. Em revisão de literatura internacional, que incluiu trabalhos realizados a partir de 1980, foram encontradas proporções de transtornos mentais que variaram de 1,0 a 51,0\% (média de 15,8\%) ${ }^{2}$.

O processo terapêutico de crianças e adolescentes com algum transtorno mental é o momento em que o paciente passa por cuidados efetivos exercidos, muitas vezes, por uma equipe multidisciplinar, tendo por finalidade o tratamento dos sintomas de sua doença e a manutenção e garantia de sua continuidade ao tratamento, visando sua melhora. É nesse momento também que deve haver uma parceria entre a equipe multiprofissional e os familiares do paciente, juntos pelo mesmo objetivo: o da melhora e qualidade de vida do paciente ${ }^{3}$.

Nesse caso, a família também é um consumidor do serviço e pode ser um aliado eficiente em conjunto com a medicação e a terapêutica trabalhada pela equipe multiprofissional. Tanto a família quanto a equipe responsável pela criança ou adolescente necessitam estar alinhadas com o objetivo de adquirir confiança e vínculo, para que se estabeleça uma relação de confiança e de aceitação do tratamento, o que garantirá a efetivação do tratamento e a estabilização do quadro ${ }^{3}$.

Em decorrência dessa maior participação da família na terapêutica, em meados da década de 1990, a OMS passou a recomendar uma abordagem avaliativa, também com a inclusão de pessoas envolvidas com o acompanhamento de pacientes, como os familiares ${ }^{1,4,5}$.

Dessa forma, uma das medidas mais utilizadas para a avaliação da qualidade do serviço é a satisfação. O conceito de satisfação tem sido formulado por diferentes modelos explicativos, sendo o mais utilizado o modelo da expectativa, segundo o qual o indivíduo avalia o serviço a partir de uma comparação que ele faz entre as expectativas que possuía previamente e os resultados reais de sua experiência ${ }^{6}$. Os fatores que contribuem para a satisfação com os serviços de saúde em geral são: características dos pacientes, como aspectos sociodemográficos e clínicos; características dos profissionais, como traços de personalidade e qualidade técnica; aspectos da relação médico-paciente; e fatores estruturais e ambientais, como tempo no serviço e frequência de atendimento ${ }^{7}$. No que diz respeito à saúde mental, os fatores associados à satisfação com os serviços ainda não foram bem-definidos, devido à escassez de estudos. Algumas pesquisas internacionais relatam associação entre satisfação e maior colaboração do serviço, maior autonomia do paciente, e menor sobrecarga do familiar ${ }^{8,9}$.

A avaliação dos familiares sobre a satisfação com os serviços de saúde mental para crianças e adolescentes pode ser particularmente útil, porque eles podem assumir a posição de principais provedores de cuidados nas atividades cotidianas, na supervisão de comportamentos problemáticos e no uso dos medicamentos, afetando os resultados do tratamento ${ }^{8,9}$. Além disso, incluir os familiares na avaliação do tratamento e conhecer os fatores que contribuem para a sua satisfação com o serviço são atitudes que podem ter um efeito positivo em seu bem-estar, contribuindo para a diminuição do grau de sobrecarga com o papel de cuidador e continuidade do tratamento ${ }^{8,10}$.

Apesar da importância dos estudos que priorizam a avaliação dos serviços em saúde mental infantojuvenil pelos familiares cuidadores, tais pesquisas ainda são escassas no Brasil ${ }^{8,10,11}$. Para isso, revisões sistemáticas são muito úteis, já que reúnem as informações de um conjunto de estudos que podem apresentar resultados contrários e/ou coincidentes, auxiliando na orientação para investigações futuras ${ }^{12}$. Portanto, o presente estudo teve como objetivo realizar uma revisão sistemática sobre a satisfação entre familiares com os serviços de saúde mental infantojuvenil, assim como analisar os fatores associados.

\section{MÉTODOS}

O método utilizado constituiu-se de uma revisão sistemática da literatura acerca dos recentes estudos que investigaram a satisfação dos familiares com os serviços de saúde mental para crianças e adolescentes. Para tal, foram utilizadas as seguintes bases de dados: PubMed/MEDLINE, ScienceDirect, Web of Science, Capes Periódicos, LILACS/BIREME, Scopus e PsycINFO, com publicações nos últimos 20 anos, em português, inglês e espanhol. Outra estratégia utilizada foi a busca manual em listas de referências dos artigos identificados e selecionados na busca eletrônica. As seleções foram tomadas com base na busca dos descritores, consultados, $a$ priori, no vocabulário Descritores de Ciências em Saúde (DeCS), por título ou resumo do artigo; na impossibilidade de clareza, os artigos foram lidos na íntegra com foque nos descritores, por dupla leitura. Todas as etapas (busca e seleção dos artigos) foram realizadas de forma independente pelos pesquisadores e as discordâncias entre eles foram resolvidas mediante discussão e consenso.

\section{Estratégia de busca}

Os descritores utilizados para a busca nas bases de dados foram: "satisfação dos consumidores", "consumer satisfaction", "satisfación de los consumidores"; "família", "family", "familia"; "criança", "child", "niño"; "adolescente", "adolescent", "adolescente"; "serviços de saúde mental", "mental health services", "servicios de salud mental" (Quadro 1).

Após a busca, realizada nas bases de dados, foi realizada uma segunda etapa para a seleção dos artigos, que deveriam preencher os seguintes critérios de inclusão: estudos epidemiológicos 
observacionais como coorte, de acompanhamento, seccional e caso-controle; estudos que utilizaram instrumentos validados para satisfação.

\section{Avaliação da qualidade dos estudos}

Para análise da qualidade dos estudos, foi utilizado o Checklist for Measuring Quality proposto por Downs e Black ${ }^{13}$, instrumento aplicável ao delineamento dos artigos para avaliação de sua qualidade, o qual permite avaliar a informação, a validade interna (vieses e confundimentos) e externa e a capacidade de detecção de efeito significativo do estudo. O presente artigo utilizou a versão composta por 27 itens, sendo excluídos os relacionados a estudos experimentais. Desse modo, ao final, foram avaliados 17 itens (Quadro 2), fase em que os artigos podiam obter até 18 pontos. Os que apresentaram classificação acima de $70 \%$ (acima de 12 pontos) foram incluídos no estudo por serem considerados pelos autores de maior rigor metodológico. Os mesmos critérios foram utilizados por outros autores em artigos de revisão nacional ${ }^{14,15}$. Nessa fase, o checklist foi aplicado de forma independente por dois pesquisadores e as discordâncias entre eles também foram resolvidas mediante discussão e consenso.

\section{Extração dos dados}

Dessa forma, os estudos que obtiveram maior pontuação foram utilizados na presente revisão e suas informações foram extraídas para comparação. As fichas para extração de dados continham as seguintes informações: autores, país e ano de publicação,

Quadro 1. Sintaxes empregadas nas respectivas bases de dados durante busca bibliográfica

\begin{tabular}{|c|c|}
\hline Bases de dados & Sintaxe empregada \\
\hline $\begin{array}{l}\text { PubMed/ } \\
\text { MEDLINE } \\
\text { ScienceDirect } \\
\text { Web of Science } \\
\text { Portal CAPES } \\
\text { Periódicos }\end{array}$ & $\begin{array}{l}\text { (("satisfação dos consumidores" AND “família” } \\
\text { AND ("criança" OR "adolescente") AND } \\
\text { "serviços de saúde mental") OR ("consumer } \\
\text { satisfaction" AND “family" AND ("child" OR } \\
\text { "adolescent") AND "mental health services") } \\
\text { OR ("satisfación de los consumidores" AND } \\
\text { "familia” AND ("niño" OR “adolescente") AND } \\
\text { "servicios de salud mental")) }\end{array}$ \\
\hline $\begin{array}{l}\text { LILACS/ } \\
\text { BIREME }\end{array}$ & $\begin{array}{l}\text { (tw:(satisfação dos consumidores)) AND } \\
\text { (tw:(família)) AND (tw:(criança)) AND } \\
\text { (tw:(adolescente)) AND (tw:(serviços de saúde } \\
\text { mental)) AND (tw:(consumer satisfaction)) } \\
\text { AND (tw:(family)) AND (tw:(child)) AND } \\
\text { (tw:(adolescent)) AND (tw:(mental health } \\
\text { services)) AND (tw:(satisfación de los } \\
\text { consumidores)) AND (tw:(familia)) AND } \\
\text { (tw:(niño)) AND (tw:(adolescent)) AND } \\
\text { (tw:(servicios de salud mental)) }\end{array}$ \\
\hline Scopus & $\begin{array}{l}\text { ALL(“consumer satisfaction") AND family AND } \\
\text { child OR adolescent AND ALL ("mental health } \\
\text { services") }\end{array}$ \\
\hline PsycInfo & $\begin{array}{l}\text { consumer satisfaction AND family AND child } \\
\text { OR adolescent AND mental health services }\end{array}$ \\
\hline
\end{tabular}

tipo de estudo, local de realização do estudo, tamanho da amostra, parentesco, diagnóstico segundo o Manual Diagnóstico e Estatístico de Transtornos Mentais (DSM-IV) ou a Classificação Internacional de Doenças e Problemas Relacionados à Saúde (CID-10), média de idade dos pacientes (crianças e adolescentes), outras variáveis investigadas pelos estudos e pontuação da avaliação qualitativa (Tabela 1), instrumento utilizado para avaliar satisfação pelos familiares, média de satisfação obtida no estudo e principais resultados (Tabela 2). Os dados foram digitados em planilha Excel.

\section{RESULTADOS}

\section{Resultados da busca}

Inicialmente, a busca bibliográfica resultou 1.114 artigos, dos quais 1.076 foram identificados por meio da busca nas bases de dados e 38, por intermédio da busca manual na seção de referências dos artigos encontrados nas bases de dados. A partir da busca dos descritores no título ou resumo ou corpo do texto, foram descartados 206, estudos por não estarem relacionados ao objetivo do presente artigo, resultando em 908 estudos. Desses, 696 foram excluídos por serem revisões de literatura, como,

Quadro 2. Itens do Checklist for Measuring Quality utilizados para a avaliação qualitativa dos artigos

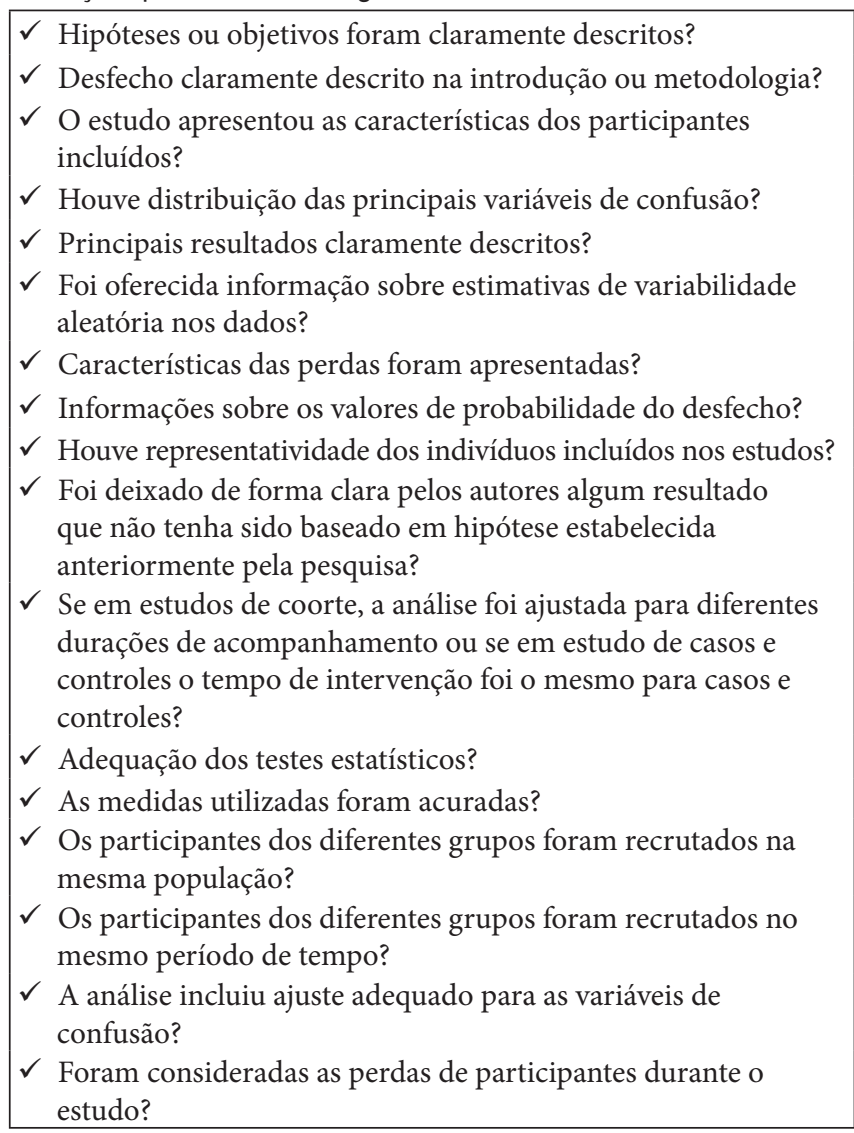




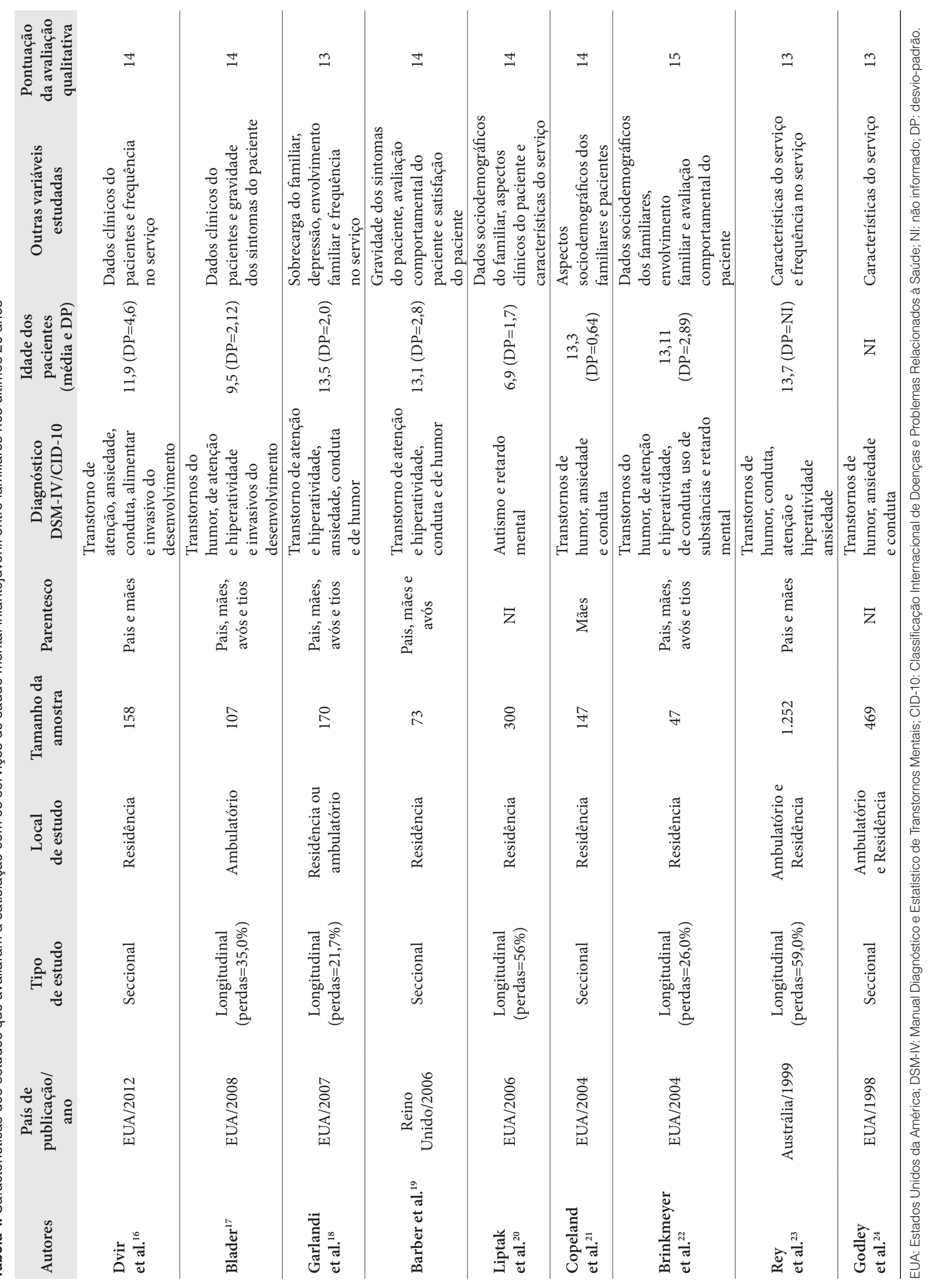


Tabela 2. Características e principais resultados dos estudos que avaliaram a satisfação com os serviços de saúde mental entre familiares de usuários nos últimos 20 anos

\begin{tabular}{|c|c|c|c|}
\hline Autores & $\begin{array}{l}\text { Escala de satisfação/ } \\
\text { pontuação da escala }\end{array}$ & $\begin{array}{l}\text { Nível de satisfação } \\
\text { global obtido/M }\end{array}$ & Principais resultados \\
\hline $\begin{array}{l}\text { Dvir } \\
\text { et al. }{ }^{16}\end{array}$ & $\begin{array}{l}\text { PSQ }(\alpha=0,96) \\
(1-5)\end{array}$ & $\begin{array}{c}\text { Satisfeito } \\
(\mathrm{M}=3,70 ; \mathrm{DP}=0,94)\end{array}$ & $\begin{array}{l}74,2 \% \text { dos familiares estiveram satisfeitos com o serviço e } 69 \% \text { encontraram o } \\
\text { que precisavam no serviço prestado à criança ou ao adolescente }\end{array}$ \\
\hline Blader $^{17}$ & $\begin{array}{l}\operatorname{CSQ}(\alpha=0,91) \\
\quad(1-4)\end{array}$ & $\begin{array}{l}\text { Muito satisfeito } \\
(\mathrm{M}=3,8 ; \mathrm{DP}=0,98)\end{array}$ & $\begin{array}{l}\text { Durante o seguimento do estudo, familiares com crianças menos graves estiveram } \\
\text { mais satisfeitos com o serviço do que os familiares com crianças mais graves }\end{array}$ \\
\hline $\begin{array}{l}\text { Garlandi } \\
\text { et al. }\end{array}$ & $\begin{array}{l}\operatorname{CSQ}(\alpha=0,91) \\
\quad(1-4)\end{array}$ & $\begin{array}{c}\text { Satisfeito } \\
(\mathrm{M}=3,20 ; \mathrm{DP}=0,55)\end{array}$ & $\begin{array}{l}\text { As variáveis que se mostraram correlacionadas à satisfação dos familiares com } \\
\text { o serviço foram menor sobrecarga }(r=-0,20 ; p<0,001) \text { e maior frequência dos } \\
\text { pacientes no serviço }(r=0,18 ; p<0,05)\end{array}$ \\
\hline $\begin{array}{l}\text { Barber } \\
\text { et al. }{ }^{19}\end{array}$ & $\begin{array}{l}\text { ESQ (alfa não } \\
\text { referido) } \\
(1-12)\end{array}$ & $\begin{array}{c}\text { Satisfeito } \\
(\mathrm{M}=10,5 ; \mathrm{DP}=\mathrm{NI})\end{array}$ & $\begin{array}{l}\text { Mais de } 90 \% \text { dos familiares estiveram satisfeitos com o serviço, em } \\
\text { comparação com os pacientes que se mostraram mais insatisfeitos }\end{array}$ \\
\hline $\begin{array}{l}\text { Liptak } \\
\text { et al. }{ }^{20}\end{array}$ & $\begin{array}{l}\text { MAPS }(\alpha=0,87) \\
\quad(1-5)\end{array}$ & $\begin{array}{c}\text { Satisfeito } \\
(\mathrm{M}=3,02 ; \mathrm{DP}=0,58)\end{array}$ & $\begin{array}{l}\text { Famílias de crianças com retardo mental estiveram mais satisfeitas com o } \\
\text { serviço do que as famílias com crianças que tinham autismo }\end{array}$ \\
\hline $\begin{array}{l}\text { Copeland } \\
\text { et al. }\end{array}$ & $\begin{array}{l}\operatorname{CSQ}(\alpha=0,91) \\
\quad(1-4)\end{array}$ & $\begin{array}{c}\text { Satisfeito } \\
(\mathrm{M}=3,18 ; \mathrm{DP}=0,82)\end{array}$ & $\begin{array}{l}\text { Famílias de cor branca apresentaram maior satisfação com o serviço }(r=0,48 \\
\mathrm{p}<0,05) \text {. Outro fator que se mostrou correlacionado à satisfação foi maior } \\
\text { idade da criança }(\mathrm{r}=0,43 ; \mathrm{p}<0,05)\end{array}$ \\
\hline $\begin{array}{l}\text { Brinkmeyer } \\
\text { et al. }{ }^{22}\end{array}$ & $\begin{array}{l}\text { IPSI }(\alpha=0,83) \\
\quad(1-5)\end{array}$ & $\begin{array}{l}\text { Muito satisfeito } \\
(\mathrm{M}=4,20 ; \mathrm{DP}=\mathrm{NI})\end{array}$ & $\begin{array}{l}\text { Familiares com menor nível de educação apresentaram maior satisfação } \\
(\mathrm{r}=-0,35 ; \mathrm{p}<0,05) \text {. Além disto, familiares mais envolvidos com o serviço } \\
\text { também estiveram mais satisfeitos }(\mathrm{r}=0,37 ; \mathrm{p}<0,05)\end{array}$ \\
\hline Rey et al. ${ }^{23}$ & $\begin{array}{l}\text { PSQ }(\alpha=0,96) \\
\quad(1-5)\end{array}$ & $\begin{array}{l}\text { Muito satisfeito } \\
(\mathrm{M}=3,80 ; \mathrm{DP}=\mathrm{NI})\end{array}$ & $\begin{array}{l}76 \% \text { dos familiares estavam satisfeitos ou muito satisfeitos com o serviço. } 69 \% \\
\text { relataram melhora no tratamento dos pacientes }\end{array}$ \\
\hline $\begin{array}{l}\text { Godley } \\
\text { et al. }{ }^{24}\end{array}$ & $\begin{array}{c}\operatorname{CSQ}(\alpha=0,91) \\
(1-4)\end{array}$ & $\begin{array}{c}\text { Satisfeito } \\
(\mathrm{M}=3,45 ; \mathrm{DP}=049)\end{array}$ & $\begin{array}{l}\text { O melhor preditor da satisfação dos familiares com o serviço foi a diminuição } \\
\text { da severidade dos sintomas apresentados pelos pacientes } \\
\text { A qualidade do atendimento individual também foi responsável por } 61 \% \text { da } \\
\text { satisfação com o serviço, enquanto a presença de atendimento familiar foi } \\
\text { responsável por } 51 \% \text { da satisfação }\end{array}$ \\
\hline
\end{tabular}

PSQ: Parent Satisfaction Questionnaire; CSQ: Client Satisfaction Questionnaire; ESQ: Experience of Service Questionnaire; MAPS: Multidimensional Assessment of Parental Satisfaction; IPSI: Inpatient Parental Satisfaction Index; $\alpha$ : alpha de Cronbach; M: média de satisfação; DP: desvio-padrão; r: correlação de Pearson.

por exemplo, capítulos de livros, ou também estudos repetidos nas bases de dados. Dos 212 restantes, 169 foram excluídos por não preencherem os critérios de inclusão. Para a análise final, 43 estudos foram avaliados qualitativamente, sendo 34 excluídos por não alcançarem o escore de qualidade desejado. Com isso, 9 artigos foram analisados na presente revisão (Figura 1).

\section{Resultados propriamente ditos}

Em todos dos estudos selecionados, os familiares estavam satisfeitos com os serviços de saúde. Seis estudos ${ }^{16,18-21,24}$ reportaram apenas satisfação, enquanto em três estudos ${ }^{17,22,23}$ o resultado global foi de muita satisfação.

Os fatores mais investigados pelos estudos foram: gravidade dos sintomas apresentados pela criança ou adolescente ${ }^{17,19,22,24}$; dados clínicos do paciente ${ }^{16,17,20}$, como diagnóstico, idade do diagnóstico, uso de medicação; frequência no serviço ${ }^{16,18,23}$; e características do serviço, como presença de atendimento familiar ${ }^{20,23,24}$. Entre os elementos associados com a satisfação, foram encontrados fatores que dizem respeito ao paciente: idade, frequência no serviço, gravidade dos sintomas e diagnóstico de retardo mental; fatores que dizem respeito ao serviço: qualidade de atendimento individual (segundo avaliação dos familiares) e presença de atendimento familiar; e fatores relacionados à família: nível educacional, cor branca dos familiares, sobrecarga e envolvimento com as atividades no serviço.
Sete estudos ${ }^{17,18,20-24}$ encontraram alguma associação entre as variáveis estudadas e a satisfação com o serviço. Apenas dois estudos ${ }^{16,19}$ não encontraram associação entre as variáveis pesquisadas e satisfação com o serviço.

\section{Características dos estudos}

Todos os estudos foram realizados em países desenvolvidos, sendo a maior parte deles concentrada nos Estados Unidos da América $(n=7)$. O desenho de estudo mais frequente também foi o longitudinal $(n=5)$, seguido pelo desenho seccional $(n=4)$. A residência foi o principal local de realização dos estudos $(n=5)$. Um estudo foi realizado em ambulatório e, em seguida, na residência do familiar $(\mathrm{n}=2)$, e um usou tanto o ambulatório como a residência. $\mathrm{O}$ número de participantes variou entre 34 e 1.252. Em relação ao grau de parentesco, a maior parte dos estudos utilizou informações tanto de pais como mães, tios e avós $(n=4)$. Apenas um estudo entrevistou somente mães.

Em relação ao diagnóstico dos pacientes, a maior parte dizia respeito ao diagnóstico de transtornos de humor e conduta $(n=7$ cada um), transtorno de atenção, hiperatividade e ansiedade ( $n=5$ cada um), e transtornos invasivos $(n=3)$. As covariáveis mais frequentes nesses estudos foram gravidade dos sintomas $(\mathrm{n}=3)$, dados clínicos dos pacientes (como diagnóstico, uso de medicação, uso de terapias alternativas) $(n=2)$, seguidos pela 


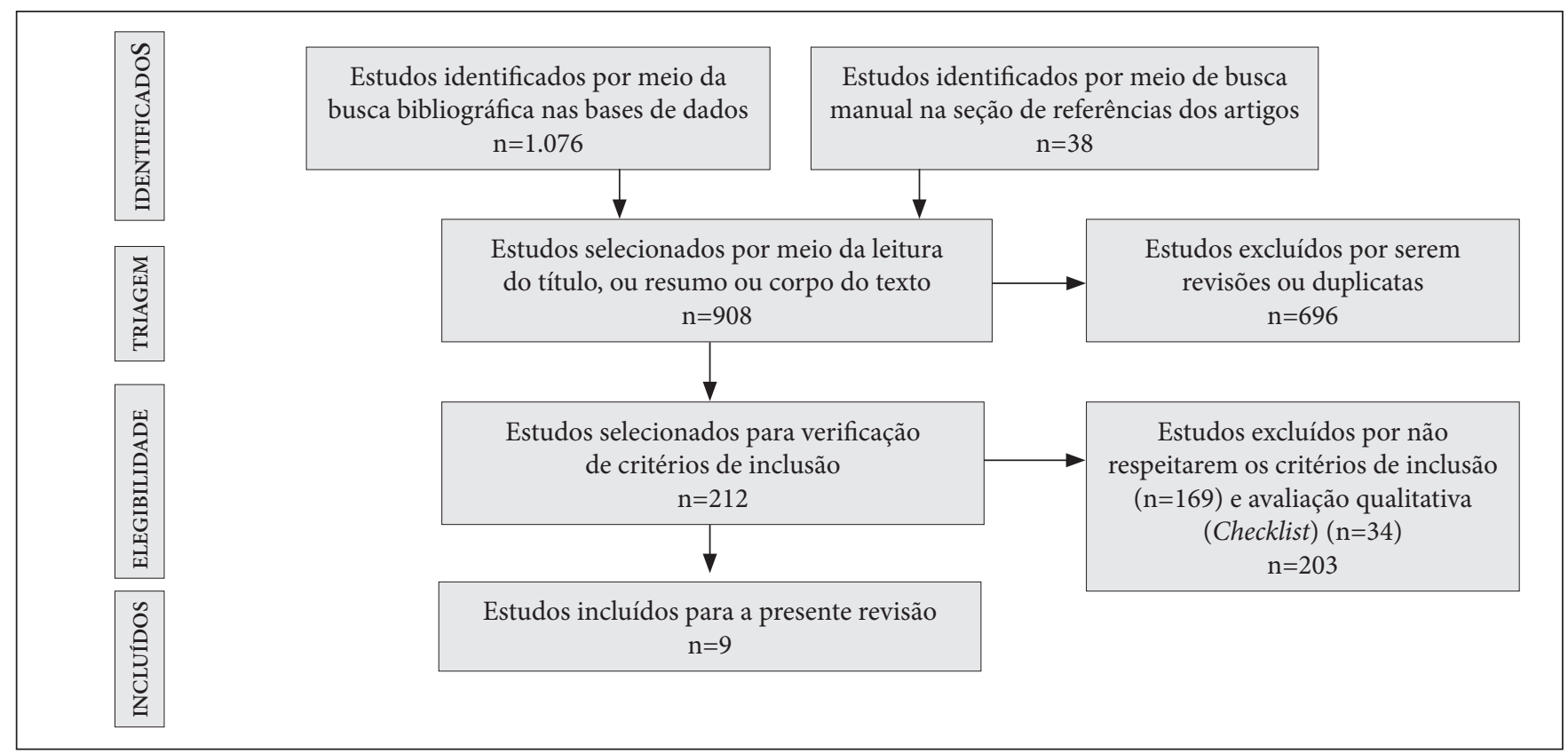

Figura 1. Fluxograma da presente revisão sistemática

frequência no serviço $(n=2)$. Em relação à idade dos pacientes, 5 estudos trabalharam com familiares de adolescentes, parcela em que a maioria possuía mais de 13 anos de idade.

Os instrumentos utilizados também foram os mais diversos, sendo os mais frequentes o Client Satisfaction Questionnaire (CSQ) ( $\mathrm{n}=4)$ e o Parent Satisfaction Questionnaire (PSQ) $(\mathrm{n}=2)$.

Em relação à qualidade metodológica dos artigos, a pontuação mínima de 13 pontos foi obtida por três estudos e o valor máximo conquistado foi 15 pontos. Apenas um estudo longitudinal ${ }^{22}$ obteve escore mais elevado em relação à pontuação máxima atribuída pelo checklist. Os vieses de seleção ou erros sistemáticos foram controlados por meio da randomização, pelo estudo de Brinkmeyer et al..$^{22}$, obtendo, assim, maior pontuação na escala metodológica. Os estudos que obtiveram menor pontuação não reportaram os valores reais de probabilidade e não relataram os principais fatores confundidores.

\section{DISCUSSÃO}

$\mathrm{Na}$ presente revisão, os estudos encontraram familiares satisfeitos com os serviços de atendimento em saúde mental infantojuvenil. Características clínicas dos pacientes, do serviço, sociodemográficas e psicossociais dos familiares estiveram associados à satisfação com o serviço de saúde mental.

Fatores como maior idade da criança e maior frequência no serviço foram identificados. Uma hipótese que tenta explicar essa relação é a de que familiares que acompanham os pacientes há mais tempo podem ter maior compreensão quanto à permanência e estabilização do quadro e menores expectativas com os serviços e, consequentemente, se declararam mais satisfeitos com $e^{2} \mathrm{e}^{25}$. Uma possível explicação para o aumento do nível de satisfação com o aumento da frequência no tratamento (duas ou três consultas semanais) é que a satisfação familiar pode depender muito da quantidade de tempo e dedicação da equipe do serviço ao paciente e sua família. Neste caso, a satisfação reflete o bom relacionamento entre a família e o serviço, em vez da melhora percebida no paciente ${ }^{23}$. Os estudos também encontraram associação entre menor gravidade dos sintomas com maior satisfação com o serviço. Marshall et al. ${ }^{26}$, em estudo longitudinal com pacientes com sintomas depressivos e doenças crônicas, constataram que doenças com maior gravidade envolvem um nível de tratamento mais complexo, o que pode dificultar a comunicação e a compreensão por parte dos familiares, prejudicando sua satisfação com o serviço.

O diagnóstico de retardo mental também se mostrou associado à satisfação com o serviço em comparação com o diagnóstico de autismo. Uma possibilidade desse contraste é que os pais de crianças com autismo podem apresentar mais dúvidas e incertezas relacionadas com a deficiência de seu filho. O estudo longitudinal de Liptak et al. ${ }^{20}$, realizado com familiares de crianças com diagnósticos de autismo e retardo mental, verificou que o sentimento de impotência pode afetar negativamente sua interação com os prestadores de cuidados de seus filhos, prejudicando a satisfação com o serviço.

Em relação aos fatores que dizem respeito ao serviço de saúde, a qualidade do atendimento individual e a presença de atendimento familiar estiveram associadas com a satisfação entre os familiares. A qualidade do atendimento individual avaliada pelo estudo de 
Godley et al. ${ }^{24}$ compreendia: a variabilidade de atendimento individual prestado (psicólogo, psiquiatra, terapeuta ocupacional), maior tempo de atendimento, melhor relação médico-paciente. No atendimento individual são atendidas as necessidades específicas do paciente, gerando na família maior segurança em relação ao serviço e, com isso, maior satisfação com o mesmo. $\mathrm{O}$ atendimento familiar é uma ferramenta muito importante no acompanhamento da assistência oferecida ao paciente, já que nesse momento as dúvidas e a carência de informação podem ser suprimidas ${ }^{27}$. Além disso, segundo Fávero ${ }^{28}$, 0 atendimento familiar pode incluir $\mathrm{o}$ atendimento em grupo, situação em que o contato com outros familiares se mostra necessário para uma troca de experiências e vivências, com o objetivo de evitar a repetição de erros e problemas.

Já em relação aos fatores relacionados às características da família, a variável cor/raça esteve associada à satisfação com o serviço. Segundo Cole et al. ${ }^{29}$, familiares de pacientes negros e de menor poder aquisitivo tendem a ser menos satisfeitos em relação aos aspectos de pós-tratamento, o que pode indicar problemas maiores em lidar com um membro doente e/ou apoio insuficiente dos serviços. Com o passar do tempo, a relação entre os serviços de saúde, pacientes negros e seus familiares começa a deteriorar-se mais do que com familiares brancos, aumentando o risco de admissão involuntária, o que, por si só, tem um impacto negativo sobre a satisfação com o atendimento. Essas diferenças configuram o racismo institucional, conceituado por Carmichael e Hamilton ${ }^{30}$ como uma falha coletiva de uma organização em prover um serviço apropriado e profissional às pessoas por causa de sua cor, cultura ou origem étnica, consequentemente minimizando os diálogos entre o paciente e o familiar e a equipe dos serviços, interferindo na autoestima do paciente e do familiar e contribuindo para sua insatisfação com os serviços ${ }^{31}$.

Outro fator associado à satisfação foi a sobrecarga familiar. O estudo de Garlandi et al. encontrou uma correlação negativa entre sobrecarga e satisfação, ou seja, quanto menor a sobrecarga, maior a satisfação com o serviço. Segundo Stegard et al., isso pode ser justificado quando a pessoa com transtorno mental faz uso da medicação de forma adequada, apresentando melhora na função social, refletindo em uma diminuição da carga do cuidador.

O baixo nível educacional também se mostrou associado com a satisfação dos familiares. Segundo $\mathrm{Neri}^{32}$, a educação apresenta uma influência decisiva sobre o estado de saúde das pessoas, já que estabelece uma relação direta com o acesso e com a reflexão sobre informações presentes. Ainda segundo $\mathrm{Neri}^{32}$, indivíduos com menor escolaridade são menos reflexivos e, consequentemente, menos críticos ao serviço, podendo, em decorrência disso, relatar maior satisfação com os serviços de saúde.

Para a investigação da satisfação e covariáveis, os instrumentos utilizados foram autopreenchíveis, do tipo escala Likert. Tal instrumento é indicado pela facilidade de administração e economia de tempo, permitindo aos respondentes divulgar um comportamento que, por constrangimento, poderia deixá-los relutantes numa entrevista face a face com o entrevistador ${ }^{33}$. Além disso, as escalas de Likert são comumente usadas em pesquisas de opinião e têm como objetivo registrar o nível de concordância ou discordância com uma declaração dada, sendo apropriadas para medir a satisfação, já que esta é uma variável que possui diferentes graus de intensidade.

Ao se trabalhar com medidas de satisfação podem ocorrer vieses que resultam em respostas excessivamente positivas, tais como o viés de gratidão e/ou de aceitação, e, no caso de análise em serviços públicos, por serem gratuitos, o receio dos familiares de perder o acesso ao serviço ${ }^{34}$. É provável que em alguns estudos os familiares tenham sido relutantes em criticar os serviços dos quais eles são tão dependentes. Desse modo, cuidados metodológicos devem ser tomados para diminuir a probabilidade desses vieses, como o uso de instruções detalhadas, destacando a importância de uma informação válida e a garantia do anonimato ${ }^{25}$. Uma forma de cuidado com os dados é o procedimento de sondagem, na qual é solicitada uma explicação para a resposta dada a cada item da escala, encorajando o entrevistado a elucidar sua compreensão do item, contribuindo, assim, para a segurança dos dados ${ }^{35}$.

De um modo geral, as perdas de acompanhamento nos estudos selecionados foram muito acentuadas. Isso pode levar à distorção das estimativas de efeito, já que constituem um importante viés de seleção, uma vez que os indivíduos perdidos no acompanhamento podem ter diferentes probabilidades de satisfação em comparação com os que permanecem no estudo, produzindo estimativas espúrias com a variável satisfação ${ }^{36}$. $\mathrm{Na}$ presente revisão, as maiores perdas foram verificadas em estudos que utilizaram residência e residência e hospital. Tais pesquisas solicitavam o retorno das respostas via Correios, o que pode ter contribuído para o elevado número de perdas.

\section{CONCLUSÃO}

Os estudos presentes nesta revisão encontraram familiares satisfeitos com os serviços de saúde mental para crianças e adolescentes. Fatores como gravidade dos sintomas, idade do paciente, frequência no serviço, diagnóstico de retardo mental, qualidade do atendimento individual, presença de atendimento familiar, nível educacional e cor branca dos familiares foram associados à satisfação com o serviço.

Desse modo, programas de assistência devem ficar atentos às medidas de satisfação, destacando a necessidade da integração da assistência em saúde mental com o familiar. Além disso, as evidências de que fatores relacionados às características dos familiares estão associados aos indicadores de satisfação levantam a importância de políticas públicas e programas na área social que invistam em educação. 


\section{REFERÊNCIAS}

1. World Health Organization. The world health report, mental health: new understanding, new hope. Geneva: WHO; 2001.

2. Roberts RE, Attkisson CC, Rosenblatt A. Prevalence of psychopathology among children and adolescents. Am J Psychiatry. 1998;155(6):715-25.

3. Lazure H. Viver a relação de ajuda: abordagem teórica e prática de critério de competência da enfermeira. Lisboa: Lusodidacta; 1994.

4. Donabedian A. Evaluating the quality of medical care. Milbank Q. 2005;83(4):691-729.

5. World Health Organization. Consumer's and caregiver's satisfaction with mental health services: a multisite study. Geneva: WHO; 1996.

6. Bandeira M, Silva MA, Camilo CA, Felício CM. Satisfação de familiares de pacientes psiquiátricos com os serviços de saúde mental e seus fatores associados. J Bras Psiquiatr. 2011;60(4):284-93.

7. Weiss GL. Patient satisfaction with primary medical care. Evaluation of sociodemographic and predispositional factor. Med Care. 1988;26(4):383-92.

8. Perreault M, Rousseau M, Provencher H, Roberts S, Milton D. Predictors of caregiver satisfaction with mental health services. Community Ment Health J. 2012;48(2):232-7.

9. Stengård EL, Honkonen T, Koivisto AM, Salokangas RK. Satisfaction of caregivers of patients with schizophrenia in Finland. Psychiatr Serv. 2000;51(8):1034-9.

10. Bandeira M, Barroso SM. Sobrecarga das famílias de pacientes psiquiátricos. J Bras Psiquiatr. 2005;54(1):34-46.

11. Camilo CA, Bandeira M, Leal RMAC, Scalon JD. Avaliação da satisfação e sobrecarga em um serviço de saúde mental. Cad Saúde Colet. 2012;20(1):82-92.

12. Linde K, Willich SN. How objective are systematic reviews? Differences between reviews on complementary medicine. J R Soc Med. 2003;96(1):17-22.

13. Downs SH, Black N. The feasibility of creating a checklist for the assessment of the methodological quality both of randomised and nonrandomised studies of health care interventions. J Epidemiol Community Health. 1998;52(6):377-84.

14. Thiengo DL, Santos JFC, Mason VC, Abelha LA, Lovisi GM. Associação entre apoio social e depressão durante a gestação: uma revisão sistemática. Cad Saúde Colet. 2011;19(2):129-38.

15. Araujo DMR, Vilarim MM, Sabroza AR, Nardi AE. Depressão no período gestacional e baixo peso ao nascer: uma revisão sistemática da literatura. Cad Saúde Pública. 2010;26(2):219-27.

16. Dvir Y, Wenz-Gross M, Jeffers-Terry M, Metz WP. "An assessment of satisfaction with ambulatory child psychiatry consultation services to primary care providers by parents of children with emotional and behavioral needs: the Massachusetts Child Psychiatry Access Project University of Massachusetts Parent Satisfaction Study". Front Psychiatry. 2012;13:7.

17. Blader JC. Longitudinal Assessment of Parental Satisfaction with Children's Psychiatric Hospitalization. Adm Policy Ment Health. 2007;34(2):108-15.

18. Garland AF, Haine RA, Boxmeyer CL. Determinates of youth and parent satisfaction in usual care psychotherapy. Eval Program Plann. 2007;30(1):45-54.

19. Barber AJ, Tischler VA, Healy E. Consumer satisfaction and child behaviour problems in child and adolescent mental health services. J Child Health Care. 2006;10(1):9-21.
20. Liptak GS, Orlando M, Yingling JT, Theurer-Kaufman KL, Malay DP, Tompkins LA, et al. Satisfaction with primary health care received by families of children with developmental disabilities. J Pediatr Health Care. 2006;20(4):245-52

21. Copeland VC, Koeske G, Greeno CG. Child and mother client satisfaction questionnaire scores regarding mental health services: race, age, and gender correlates. Res Soc Work Pract. 2004;14(6): 43442 .

22. Brinkmeyer MY, Eyberg SM, Nguyen ML, Adams RW. Family Engagement, Consumer Satisfaction and Treatment Outcome in the New Era of Child and Adolescent In-Patient Psychiatric Care. Clin Child Psychol Psychiatry October. 2004;9(4):553-66.

23. Rey JM, Plapp JM, Simpson PL. Parental satisfaction and outcome: a 4-year study in a child and adolescent mental health service. Aust N Z J Psychiatry. 1999;33(1):22-8.

24. Godley SH, Fiedler EM, Funk RR. Consumer satisfaction of parents and their children with child/adolescent mental health services. Evaluation and Program Planning. 1998;21(1):31-45.

25. Mercier C, Corten P. Évaluation de la qualité de vie de patients psychotiques. In: Kovess V, editor. Evaluation de la qualité en psychiatrie. Paris: Economica; 1994.

26. Marshall GN, Hays RD, Mazel R. Health status and satisfaction with health care: results from the medical outcomes study. J Consult Clin Psychol. 1996;64(2):380-90.

27. Contim D, Chaud MN, Fonseca AS. As questões familiares e a criança e o adolescente com doença crônica: um estudo de revisão bibliográfica. Nursing (São Paulo). 2005;8(85):267-71.

28. Fávero MAB, Santos MA. Autismo infantil e estresse familiar: uma revisão sistemática da literatura. Psicol Reflex Crit. 2005;18(3): $358-69$.

29. Cole E, Leavey G, King N, Johnson-Sabine E, Hoar A. Path ways to care for patients with first episode of psychosis. A comparison of ethnic groups. Br J Psychiatry. 1995;167(6):770-6.

30. Carmichael S, Hamilton C. Black power: the politics of liberation in America. New York: Vintage Books; 1967.

31. Silva ML. Racismo e os efeitos na saúde mental. In: Batista LE, Kalckmann S, organizador. Seminário saúde da população negra estado de São Paulo, 2004. São Paulo: Instituto de Saúde; 2005. p. 129-32.

32. Neri M, Soares W. Desigualdade social e saúde no Brasil. Cad Saúde Pública. 2002;18(Suppl):77-87.

33. Susser M. What is a cause and how do we know one? A grammar for pragmatic epidemiology? Am J Epidemiol. 1991;133(7):635-48

34. Esperidião M, Trad LAB. Avaliação da satisfação de usuários. Ciênc Saúde Coletiva. 2005;10(Suppl):303-12.

35. Guillemin F, Bombardier C, Beaton D. Cross-cultural adaptation of health-related quality of life measures: literature review and proposed guidelines. J Clin Epidemol. 1993;46(12):1417-32.

36. Reichenheim ME, Moraes CL. Alguns pilares para a apreciação da validade de estudos epidemiológicos. Rev Bras Epidemiol. 1998;1(2): 131-48. 